\title{
The influence of mandibular divergence on facial soft tissue thickness in class I patients: a cephalometric study
}

\author{
T.M. Perović1, 2®D, M. Blažej', I. Jovanović1 \\ ${ }^{1}$ Faculty of Medicine, University of Niš, Niš, Serbia \\ 2Dental Clinic, Department for Orthodontics, Niš, Serbia \\ ${ }^{3}$ Private Dental Clinic, Smiledent, Niš, Serbia
}

[Received: 12 January 2021; Accepted: 3 March 2021; Early publication date: 22 March 2021]

Background: The aims of this study were to evaluate the association between mandibular divergence and facial soft tissue thickness (FSTT) measured at different profile levels, and the gender difference in FSTT.

Materials and methods: Lateral cephalograms were used to examine nine linear distances: the glabella area (G-G1), nasal (N-N1) and subnasal area (A-Sn), upper (Sd-Ls) and lower lip thickness (Id-Li), mentolabial sulcus (B-Sm), chin area (Pg-Pg1), gnathion area (Gn-Gn1) and menton area (Me-Me1) in 155 adult Caucasian subjects (79 males, 76 females) from the central Balkan area. Subjects were divided into three groups according to the ANB angle, Wit's appraisal and SN/ GoGn angle into normodivergent (28 male, 27 female subjects), hypodivergent (26 males, 25 females) and hyperdivergent (25 males, 24 females).

Results: Progressive decreasing in the soft tissue thickness from hypo- towards hyperdivergent group was established in N-N1, A-Sn, Gn-Gn1, Me-Me1. There are significant differences in Gn-Gn1 and Me-Me1 $(p<0.02)$. Progressive increasing of FSTT happens only at the level of mentolabial sulcus and these differences are significant. Significant gender differences were established for the following distances: N-N1 in hyperdivergent, A-Sn in all three examined groups, the upper lip thickness in normo- and hyperdivergent, the lower lip thickness in hypodivergent, the thickness of mentolabial sulcus in hypo- and normodivergent, Pg-Pg1 in hyperdivergent and Me-Me1 in normodivergent subjects $(p<0.05)$.

Conclusions: Facial soft tissue thickness showed a various degree of dependence on vertical developmental pattern at different levels of measurement. The areas whose thickness is significantly conditioned by this pattern were established: the chin area at level Gn-Gn1, Me-Me1 and the region of the mentolabial sulcus $(B-S m)$. At most levels, male subjects have thicker soft tissues and these differences are significant for all three groups in the subnasal area. (Folia Morphol 2022; 81, 2: 472-480)

Key words: mandibular divergence, soft tissue thickness, face 


\section{INTRODUCTION}

Facial contours are traditionally considered to be a result of the position of basic dentoskeletal tissue followed by soft tissue [19]. However, a contemporary approach shows a change in this attitude in terms of variability of the thickness of the covering soft tissue, which does not only passively follow the bone tissue. The covering soft tissues of the face (skin, fat and muscles) can develop proportionately or disproportionately relative to the corresponding skeletal structures. Variations can include thickness, length and the tone of soft tissue and they are conditioned by the sex, age, race and ethnicity, as well as the growth pattern [17, 22-24].

The influence of sagittal developmental pattern on the facial soft tissue thickness (FSTT) was proven in multiple contemporary studies. Increased soft tissue thickness was reported where there is anteroposterior skeletal jaw deficiency $[2,18]$. Where there is not a jawbone deficiency, there are the greatest gender differences in the soft tissue thickness [16].

However, according to the latest knowledge, vertical pattern of growth also affects the relationship between the bone tissue and covering soft tissue. According to the type of mandibular divergence, faces can be hyperdivergent (high angle-mandibular clockwise rotation) (Fig. 1), hypodivergent (low angle-mandibular counterclockwise rotation) (Fig. 2) and average, normodivergent faces (normal angle) (Fig. 3).

Developmental changes of cranial base and mandibular ramus with condyle and gonial angle determine the direction in which vertical face development will dominate. The characteristics of hyperdivergent growth are increased gonial angle, retroflexion of condyles in relation to mandibular ramus, decreasing in the length of the back part of cranial plane and the decreasing of the angle of cranial base. This excessive vertical growth may result in a gummy smile, lip incompetence and elongated face. In hypodivergent growth, these changes are reverse. There is a lack of vertical growth which can lead to the excessive exposure of incisors, deep bite and the reduced lower third of the face $[1,5,25]$. In normal divergence growth, vertical face growth is harmonious in relation to the growth in other directions.

The behaviour of soft tissue in relation to mandibular divergence pattern was mostly researched in the chin area. Therefore, decreased chin prominence in vertical growth pattern was established; in horizontal growth pattern there is a normal or increased chin

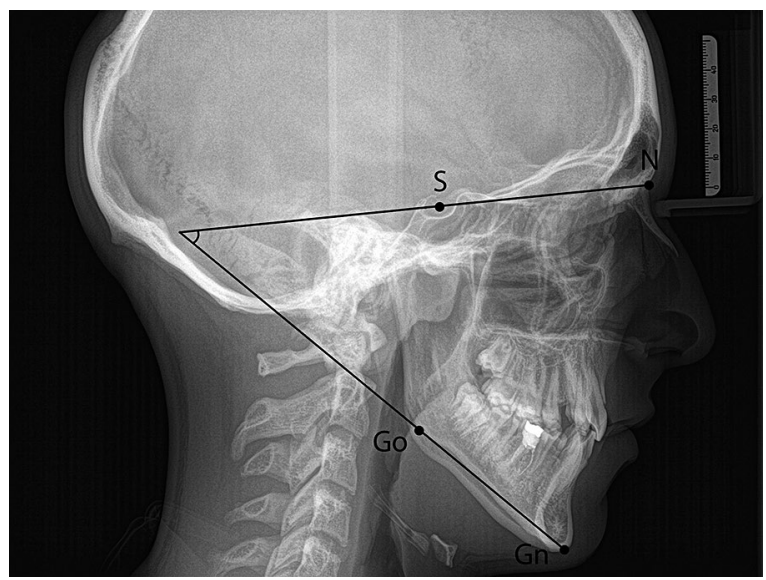

Figure 1. Lateral cephalogram of hyperdivergent pattern.

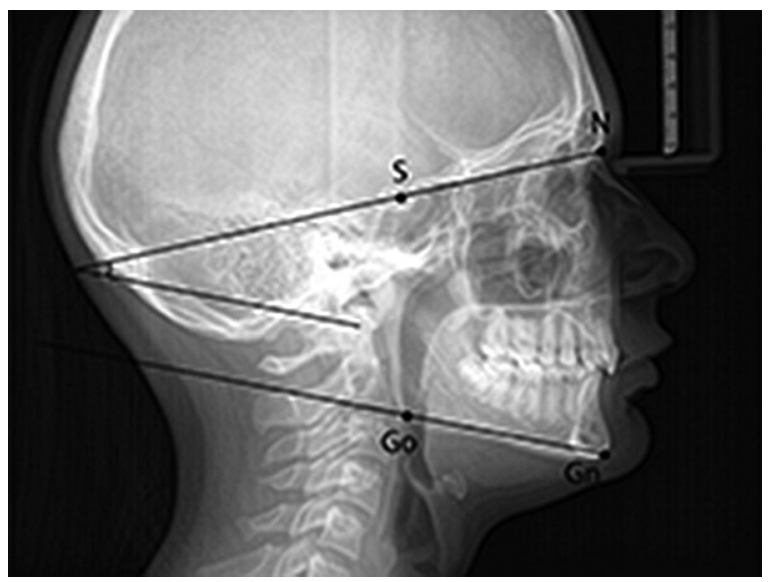

Figure 2. Lateral cephalogram of hypodivergent pattern.

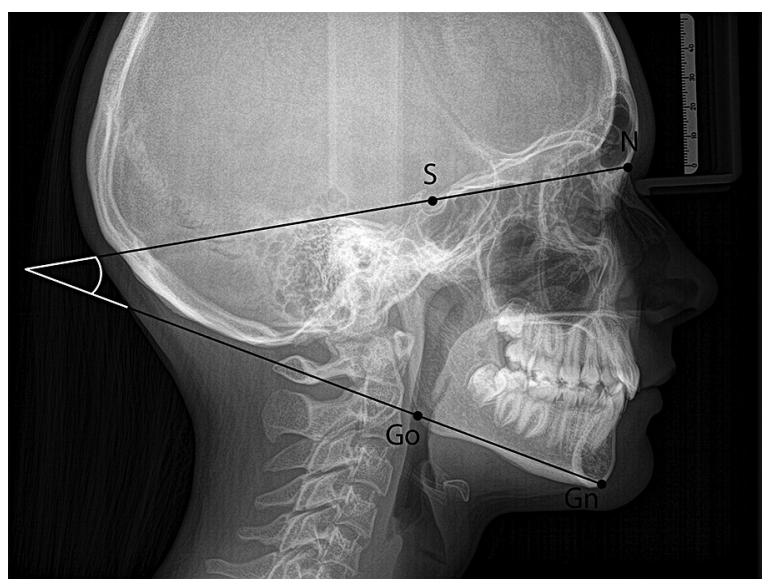

Figure 3. Lateral cephalogram of normodivergent pattern.

prominence by virtue of the mandibular counterclockwise rotation [6]. According to Shinde et al. [21], soft 
tissue chin thickness adjusts to the position of skeletal chin and it is different at various levels of the chin. Divergent patterns of the mandible not only affect the soft tissue chin thickness, but they can cause changes in the length and thickness of the upper lip [6].

The aims of this study were to evaluate the following in adult patients: 1) the association between mandibular divergence and FSTT measured at different profile levels and 2) the difference in FSTT between males and females.

\section{MATERIALS AND METHODS}

A cross-sectional, comparative, descriptive clinical study was undertaken, which was approved by the Faculty of Medicine in Niš under the general project title of Clinical and Experimental Examination of the Stomatognathic System and Modern Therapeutic Procedures, Project Number 11, from March 8, 2017, Niš, Republic of Serbia. All patients provided written informed consent to participate in the study.

This study included the examination and the analysis of cephalometric radiography-derived lateral cephalograms to evaluate FSTT for 155 adult Caucasian orthodontic patients ( 79 males, 76 females) from the mid-Balkan region, which were taken from the patient archives. Cephalometric radiography-derived lateral cephalograms were recorded during routine diagnostic procedures for patients who were examined in the Department of Jaw Orthopaedics at the Clinic of Dentistry in Niš, who were aged between 18 and 22 years, and who underwent orthodontic therapy for the first time. Patients were excluded from the study if they had a history of trauma, craniofacial anomalies, cleft lip and palate, forced bite and previous orthodontic, prosthetic or orthognathic surgical treatment.

All patients included in the study underwent a detailed clinical assessment and analysis of their dental and skeletal profiles, as well as soft tissue profiles on cephalometric radiography. The equipment used for the imaging analysis was the Rotograf Plus (20090 Buccinasco MI Italy) (number and series: 00036045), and the CEI-OPX/105 X-ray tube (CEI, Bologna) in March 2000, which had a protective filter $(2.5 \mathrm{~mm}$ aluminum-equivalent). Lateral cephalometric films were taken from a distance of $165 \mathrm{~cm}$ from the tube, using a cephalostat to ensure rigid head fixation (head-holding device).

The patients were placed in the cephalostat in such a way that the sagittal plane of the head was at a $90^{\circ}$ angle to the path of the X-rays. The Frankfort horizontal plane (it connects the upper edge of the external auditory orifice and the lowest point of the infraorbital edge) was parallel to the ground, the teeth were in a central occlusion position, and the lips were in a relaxed position. Each cephalogram was fixed on the viewing box with the profile to the right, and the acetate tracing paper was fixed by tape at the top. The soft tissue and skeletal features were traced manually in a darkened room, using a $0.5 \mathrm{~mm}$ lead pencil. All the image tracing was done by the main investigator.

Based on the values of ANB angle, Wit's appraisal, SN/GoGn angle, 155 cephalograms were finally chosen for the study and three study groups were formed. The cephalometric ANB angle (the points that determined the ANB angle included point ' $\mathrm{N}$ ', the nasion, located on the suture between the frontal and nasal bones; point ' $A$ ', the lowest point on the line between the anterior nasal spine and the prosthion [alveolar point]; and point ' $\mathrm{B}$ ', the lowest point from the line between the infradentale and the pogonion [midline of the chin]) was the parameter that defined the mutual sagittal relationship between the upper and lower jaw as orthognathic $\left(1^{\circ} \leq \mathrm{ANB} \leq 3^{\circ}\right)$ and a Wit's appraisal $\pm 1 \mathrm{~mm}$ were categorised as skeletal class I. Wit's appraisal was used to overcome the limitations of the ANB angle and entails drawing perpendiculars from points $A$ and $B$ onto the occlusal plane. The results of the Wit's appraisal were evaluated in order to eliminate the possibility of the mandibular posterior rotation obscuring any skeletal anomalies in the patients with increased vertical direction values. For this purpose, the radiographs were excluded if the results from the ANB angle measurements and Wit's appraisal did not coincide (Fig. 4).

S-N/Go-Gn - angle formed by lines S-N and Go-Gn and allows for the identification of relationship between the mandibular plane and the cranial base. It indicates the mandibular vertical developmental trend as it identifies the direction of mandibular growth rotation (Fig. 5).

According to this angle, the types are divided into hypodivergent group (angle value less than $26^{\circ}$ in 26 male, 25 female subjects), normodivergent group (angle value between $26^{\circ}$ and $38^{\circ}$ in 28 males, 27 females) and hyperdivergentgroup (angle value greater than $38^{\circ}$ in 25 males, 24 females).

Each cephalogram was checked for its magnification and dealt with accordingly. Then, the soft tissue thick- 


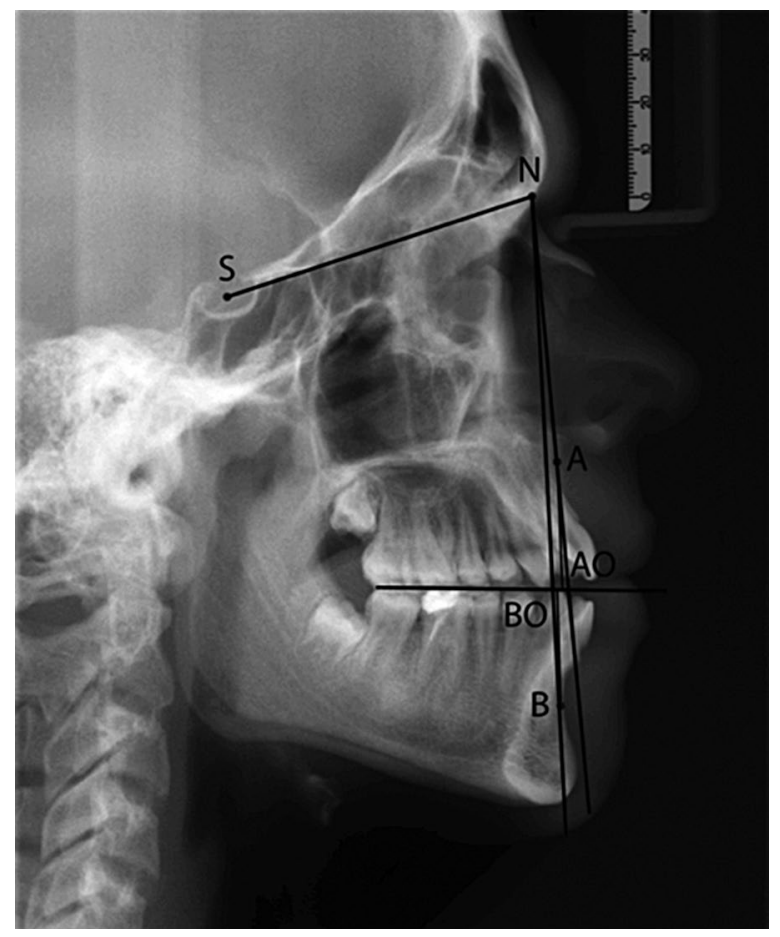

Figure 4. Cephalometric planes and angles: ANB angle and Wit's appraisal for the identification of mutual sagittal jaw relationship.

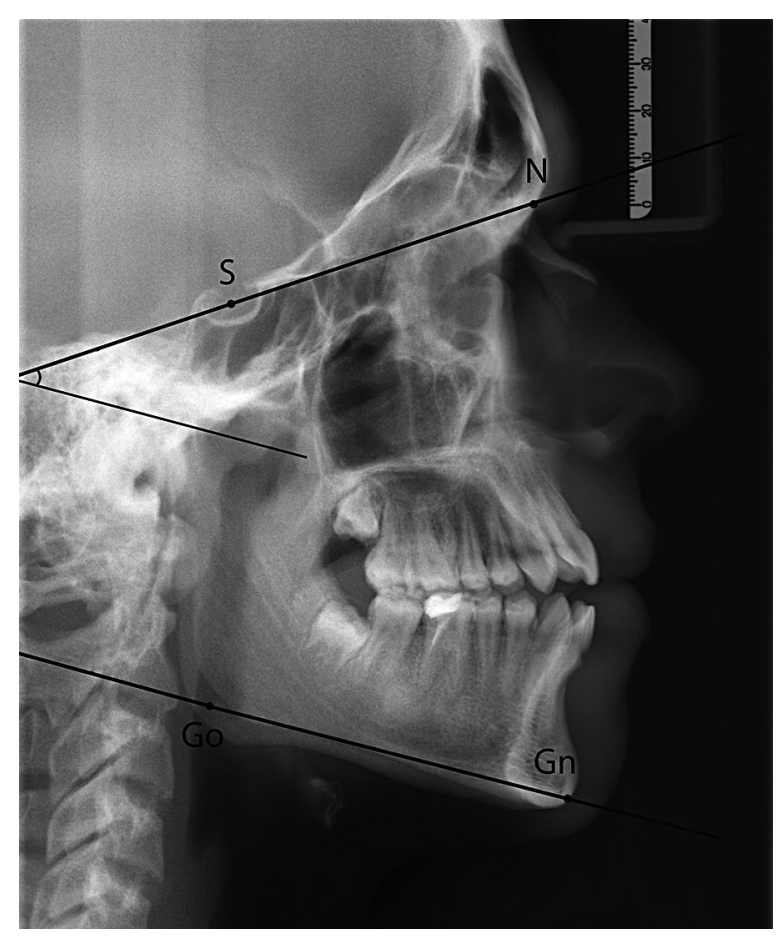

Figure 5. Cephalometric S-N/Go-Gn angle for the identification of mandibular divergence pattern.

ness was measured for each cephalogram at nine selected distances (Fig. 6). The following areas were used:

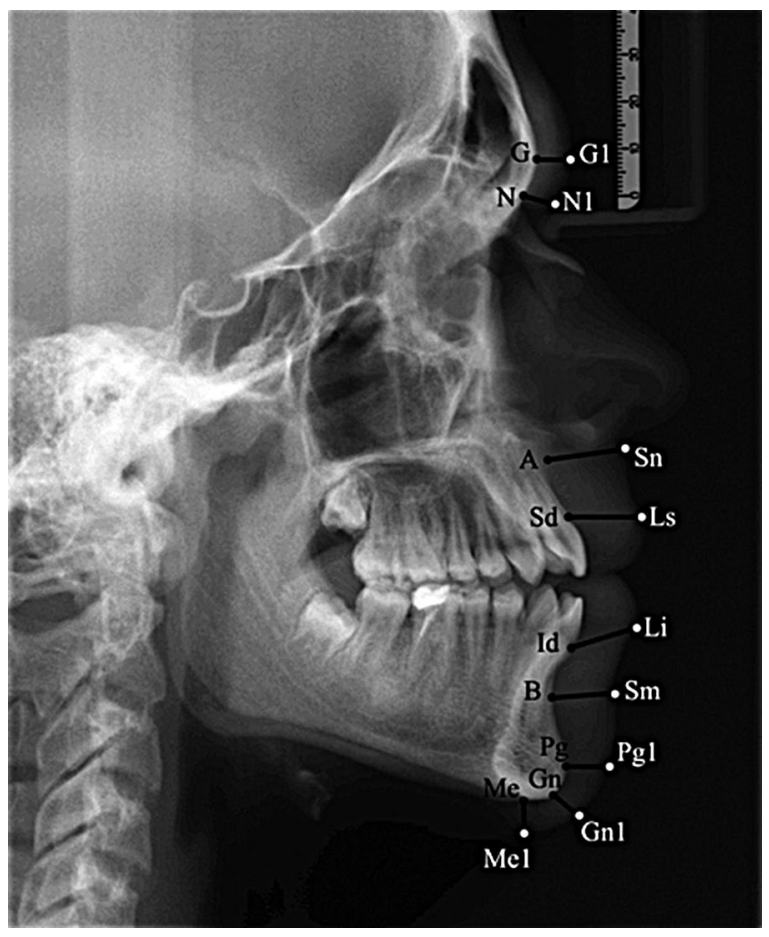

Figure 6. Nine soft tissue cephalometric landmarks (from top to bottom): G-G1, N-N1, A-Sn, Sd-Ls, Id-Li, B-Sm, Pg-Pg1, Gn-Gn1, Me-Me1.

- the glabella area (G-G1) - the linear distance between the $\mathrm{G}$ point (the most prominent point on the frontal bone) and the soft tissue, or analogue point (G1);

- the nasal area (N-N1) - the linear distance between the $\mathrm{N}$ point and on the soft tissue, the deepest point of the root of the nose (N1);

- the subnasal area (A-Sn) - the distance between point $A$ (the most concave point of the anterior maxilla) and subnasale (the point at which the nasal septum merges with the upper lip);

- upper lip thickness (Sd-Ls) - the distance between the Sd point (supradentale, prosthion - the most inferior anterior point on the maxillary alveolar process between the central incisors) and the Ls (labrale superius - the most anterior point on the upper lip);

- lower lip thickness (Id-Li) — the distance between the Id point (infradentale - the highest point of the mandibulary alveolar process between the two central incisors) and Li point (labrale inferius - the most anterior point on the lower lip);

- the sulcus mentolabialis (B-Sm), the distance between the $B$ point (the most concave point on mandibular symphysis) and Sm (mentolabial sulcus - the point on greatest concavity in the midline between the labrale inferius and soft tissue pogonion); 
Table 1. Median value of facial soft tissue thickness in subjects with different mandibular divergence pattern and group differences

\begin{tabular}{|c|c|c|c|c|c|c|c|c|c|c|c|c|}
\hline & \multicolumn{4}{|c|}{ Hypodivergent } & \multicolumn{4}{|c|}{ Normodivergent } & \multicolumn{4}{|c|}{ Hyperdivergent } \\
\hline & $\mathbf{N}$ & Median & $25 \%$ & $75 \%$ & $\mathbf{N}$ & Median & $25 \%$ & $75 \%$ & $\mathbf{N}$ & Median & $25 \%$ & $75 \%$ \\
\hline G-G1 & 51 & 6.00 & 5.68 & 6.15 & 55 & 6.20 & 5.69 & 6.63 & 49 & 5.85 & 5.47 & 6.85 \\
\hline N-N1 & 51 & 6.78 & 6.15 & 6.97 & 55 & 6.53 & 5.72 & 7.15 & 49 & 5.88 & 5.34 & 7.24 \\
\hline A-Sn & 51 & 15.99 & 13.30 & 16.35 & 55 & 14.91 & 13.70 & 16.69 & 49 & 14.58 & 13.16 & 16.35 \\
\hline Sd-Ls & 51 & 14.82 & 13.86 & 15.63 & 54 & 15.14 & 13.44 & 16.70 & 49 & 14.59 & 12.60 & 16.93 \\
\hline Id-Li & 51 & 15.25 & 14.44 & 16.68 & 55 & 15.91 & 14.34 & 17.08 & 49 & 15.60 & 14.28 & 17.21 \\
\hline B-Sm & 51 & 10.44 & 9.84 & 10.99 & 55 & $11.03^{b}$ & 10.10 & 12.10 & 49 & $11.61^{\mathrm{d}}$ & 10.18 & 12.63 \\
\hline $\mathrm{Pg}-\mathrm{Pg} 1$ & 51 & 11.08 & 10.00 & 13.97 & 55 & 12.14 & 10.73 & 13.68 & 49 & 11.27 & 9.76 & 13.18 \\
\hline Gn-Gn1 & 51 & $9.80^{d}$ & 8.09 & 11.12 & 55 & 9.22 & 7.76 & 10.72 & 49 & 7.31 & 6.26 & 9.65 \\
\hline Me-Me1 & 51 & $8.29^{d}$ & 6.46 & 9.62 & 55 & $7.91^{c}$ & 6.75 & 8.81 & 49 & 7.10 & 5.49 & 8.67 \\
\hline
\end{tabular}

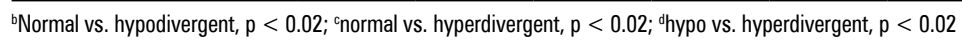

Table 2. Results of Kruskal-Wallis ANOVA test of the facial soft tissue thickness of groups with different mandibular divergence pattern

\begin{tabular}{lccccccccc}
\hline & G-G1 & N-N1 & A-Sn & Sd-Ls & Id-Li & B-Sm & Pg-Pg1 & Gn-Gn1 & Me-Me1 \\
\hline Kruskal-Wallis H & 2.838 & 3.407 & 0.429 & 1.243 & 1.576 & 13.768 & 2.635 & 15.412 & 8.236 \\
df & 2 & 2 & 2 & 2 & 2 & 2 & 2 & 2 & 2 \\
Asymp. Sig. & 0.242 & 0.182 & 0.807 & 0.537 & 0.455 & 0.001 & 0.268 & $<0.001$ & 0.016 \\
\hline
\end{tabular}

- the chin area $(\mathrm{Pg}-\mathrm{Pg} 1)$ - the distance between the Pg point (the pogonion - the most prominent point of the chin), and soft tissue pogonion Pg1 (the most anterior point on the soft tissue chin);

- the gnathion area (Gn-Gn1) - the distance between bony $\mathrm{Gn}$ (gnathion - the lowest point on the anterior margin of the lower jaw in the midsaggital plane) and soft tissue Gn1 (the constructed midpoint between soft tissue pogonion and soft tissue menton);

- the menton area (Me-Me1) - the distance between the Me (menton - at the junction between the mandibular symphyseal outline and the inferior border of the mandibular body) and Me1 point (soft tissue menton - lowest point on the contour of the soft tissue chin).

The values of the soft tissue thickness were measured with a digital calliper (in millimetres). All the measurements were randomly done once by an experienced orthodontist (principal investigator). Nine linear distances analysed statistically in the three groups of subjects and categorised according to gender. The median values in males and females were compared in each group of subjects with different types of divergence.

\section{Statistical method}

Statistical analysis was performed by IBM SPSS statistical package (version 23). Significance of differences between analysed groups was analysed by Kruskal-Wallis $\mathrm{H}$ test. Detected significant differences were additionally analysed by Mann-Whitney $U$ test with $p$ values modified according to Bonferroni correction $(p<0.02)$. Significance of gender differences in analysed groups were evaluated by Mann-Whitney $\mathrm{U}$ test.

\section{RESULTS}

The values of the examined parameters, compared to vertical pattern and gender, are presented in Tables 1,2 , and 3 .

Group differences. Progressive decreasing of the soft tissue thickness from hypo- towards hyperdivergent group was established in N-N1, A-Sn, Gn-Gn1, Me-Me1. There are significant differences in $\mathrm{Gn}-\mathrm{Gn} 1$ and Me-Me1 $(p<0.02)$. Progressive increasing of the soft tissue thickness happens only at the level of mentolabial sulcus and these differences are significant (Tables 2, 3).

Gender differences. For the greatest number of the examined distances, there is a rule that the 
Table 3. Descriptive statistics of the facial soft tissue thickness linear parameters in male and female subjects with different mandibular divergence pattern

\begin{tabular}{|c|c|c|c|c|c|c|c|c|c|c|c|c|}
\hline \multirow[t]{2}{*}{ Gender } & \multicolumn{4}{|c|}{ Hypodivergent } & \multicolumn{4}{|c|}{ Normodivergent } & \multicolumn{4}{|c|}{ Hyperdivergent } \\
\hline & $\mathbf{N}$ & Median & $25 \%$ & $75 \%$ & $\mathbf{N}$ & Median & $25 \%$ & $75 \%$ & $\mathbf{N}$ & Median & $25 \%$ & $75 \%$ \\
\hline \multicolumn{13}{|l|}{ Male } \\
\hline G-G1 & 26 & 5.98 & 5.85 & 6.19 & 28 & 6.08 & 5.65 & 6.47 & 25 & 5.94 & 5.51 & 7.08 \\
\hline N-N1 & 26 & 6.73 & 6.15 & 8.11 & 28 & 6.73 & 5.90 & 7.21 & 25 & $6.39^{a}$ & 5.70 & 7.54 \\
\hline $\mathrm{A}-\mathrm{Sn}$ & 26 & $16.34^{\mathrm{a}}$ & 16.23 & 16.41 & 28 & $16.02^{\mathrm{a}}$ & 14.91 & 17.29 & 25 & $16.00^{\mathrm{a}}$ & 14.73 & 16.82 \\
\hline Sd-Ls & 26 & 15.36 & 14.51 & 15.63 & 28 & $16.13^{\mathrm{a}}$ & 15.05 & 17.45 & 25 & $16.15^{\mathrm{a}}$ & 14.71 & 18.29 \\
\hline Id-Li & 26 & $16.24^{a}$ & 15.46 & 17.89 & 28 & 16.22 & 14.81 & 17.17 & 25 & 16.46 & 14.82 & 18.12 \\
\hline B-Sm & 26 & $10.68^{a}$ & 10.15 & 11.45 & 28 & $11.33^{a}$ & 10.84 & 12.77 & 25 & 11.62 & 11.08 & 13.14 \\
\hline $\mathrm{Pg}-\mathrm{Pg} 1$ & 26 & 12.62 & 9.41 & 14.40 & 28 & 12.25 & 10.84 & 13.71 & 25 & $11.90^{\mathrm{a}}$ & 10.49 & 13.30 \\
\hline Gn-Gn1 & 26 & 9.06 & 7.93 & 12.39 & 28 & 9.65 & 8.25 & 10.89 & 25 & 7.76 & 6.76 & 10.24 \\
\hline Me-Me1 & 26 & 8.47 & 6.22 & 9.62 & 28 & $8.15^{a}$ & 7.40 & 9.75 & 25 & 7.13 & 5.88 & 9.82 \\
\hline \multicolumn{13}{|l|}{ Female } \\
\hline G-G1 & 25 & 6.00 & 5.49 & 6.15 & 27 & 6.23 & 5.86 & 6.78 & 24 & 5.71 & 5.36 & 6.67 \\
\hline N-N1 & 25 & 6.78 & 5.38 & 6.97 & 27 & 6.43 & 5.71 & 6.98 & 24 & 5.59 & 5.13 & 6.50 \\
\hline $\mathrm{A}-\mathrm{Sn}$ & 25 & 14.30 & 11.45 & 15.64 & 27 & 14.17 & 12.61 & 14.62 & 24 & 13.36 & 11.81 & 14.54 \\
\hline Sd-Ls & 25 & 13.91 & 10.59 & 15.20 & 27 & 13.85 & 12.45 & 15.22 & 24 & 13.16 & 11.93 & 14.49 \\
\hline Id-Li & 25 & 14.64 & 13.49 & 14.99 & 27 & 15.17 & 14.12 & 16.81 & 24 & 15.15 & 13.69 & 16.15 \\
\hline B-Sm & 25 & 10.27 & 9.47 & 10.72 & 27 & 10.58 & 9.90 & 11.49 & 24 & 10.90 & 9.92 & 12.20 \\
\hline $\mathrm{Pg}-\mathrm{Pg} 1$ & 25 & 10.87 & 10.16 & 11.27 & 27 & 11.59 & 9.95 & 13.51 & 24 & 10.63 & 9.03 & 12.88 \\
\hline Gn-Gn1 & 25 & 9.80 & 8.45 & 11.12 & 27 & 8.64 & 6.81 & 10.30 & 24 & 6.90 & 5.75 & 8.38 \\
\hline
\end{tabular}

Males vs. females, $\mathrm{p}<0.05$

larger values of the soft tissue thickness were recorded in males. The exception is G-G1 in normal and hypodivergent subjects; N-N1 and Gn-Gn1 in hypodivergent subjects, where the larger values were established in females. Significant gender differences were established for the following distances: N-N1 in hyperdivergent, A-Sn in all three examined groups, the upper lip thickness in normal and hyperdivergent, the lower lip thickness in hypodivergent, the thickness of mentolabial sulcus in hypo- and normodivergent, $\mathrm{Pg}-\mathrm{Pg} 1$ in hyperdivergent and Me-Me1 in normodivergent subjects ( $p<0.05$; Table 1$)$.

\section{DISCUSSION}

The basic issue in this research was, how do the covering soft tissues adjust to the mandibular divergence? Do they passively follow the bone base, so that by 'elongating' the facial skeleton soft tissues become thinner? Or, similar to sagittal developmental pattern, soft tissues compensate for the vertical disharmony with their thickness? Ajwa et al. [2] and Jazmati et al. [10] think that variations in the soft tissue thickness are not correlated with craniofacial morphology. Kamak and Celikoglu [12] established that there are only differences in the lip area.

In general, the majority of studies reported that male subjects had thicker soft tissue than female subjects with the variable degree of significance. In the present study, there are levels at which female subjects have thicker soft tissues such is the level G-G1, for example, but these differences are not significant. Furthermore, the differences between various groups of divergence at G-G1 level do not show significance or a clear tendency of changes in thickness, going from hypo- towards hyperdivergent group. At level $\mathrm{N}-\mathrm{N} 1$, there is already a slightly pronounced tendency of decreasing of soft tissue thickness. This phenomenon is more conspicuous in females, but without statistical relevance among groups. Significant gender differences were established only in hyperdivergent group. According to Al Mashadany et al. [4], the soft tissues thickness in glabella in males is insignificantly larger in hypodivergent group. The majority of group differences were established between hypoand hyperdivergent groups, which was confirmed by our study as well. On the other hand, the same 
author recorded significant difference at level N-N1, especially between hypo- and hyperdivergent group.

At level A-Sn, progressive decreasing in soft tissue thickness happens, from hypo- towards hyperdivergent pattern. This phenomenon is established in both gender, but group differences are not significant. However, Khare and Niwlikar [13] reported that subjects with hyperdivergent growth will develop thicker soft tissue at this level, which is opposite to the current study. Males at this level had larger soft tissue thickness than females and these differences are significant for all three groups of divergence.

In the present study, the thickness of the upper and lower lip differs slightly among the groups of various divergences, and there are significant gender differences for the upper lip in normal and hyperdivergent group, and for the lower lip in hypodivergent group in favour of males. According to Al Sajagh et al. [3], hyperdivergent females exhibited significantly larger lower lip thickness compared to the other two types of face, which was not the case in the present study - the average values in normal and hyperdivergent females differ insignificantly. In normodivergent males there is significantly larger upper lip thickness at level A-Sn and Sd-Ls, as well as for lower lip thickness Id-Li, compared to female subjects. The upper lip thickness at point A-Sn and $\mathrm{Sd}-\mathrm{Ls}$, and lower lip thickness in Li were significantly larger in males than in females [3], which is also similar to our results. Celikoglu et al. [7] established only in females smaller values of the thickness of the upper and lower lip of statistical relevance in hyperdivergent group compared to the values in normodivergent group. Furthermore, hypo- and normodivergent groups showed similar values of thickness. In males, there were not any statistically significant differences among various mandibular divergence patterns [7], which is similar to our results. Khatri and Sanap [14] established larger lower lip thickness in hyperdivergent subjects in comparison to the hypodivergent, except from the fact that he examined subjects with skeletal class II. Feres et al. [9] established that soft tissue thickness of the upper and lower lip shows no differences in all morphological groups, whereas Ashraf et al. [6] established significant difference in the upper lip thickness only between hypo- and hyperdivergent group. The larger values for the lip thickness were noticed in the hypodivergent group. It was determined that the difference in the upper lip thickness is statistically significant among the three examined groups.

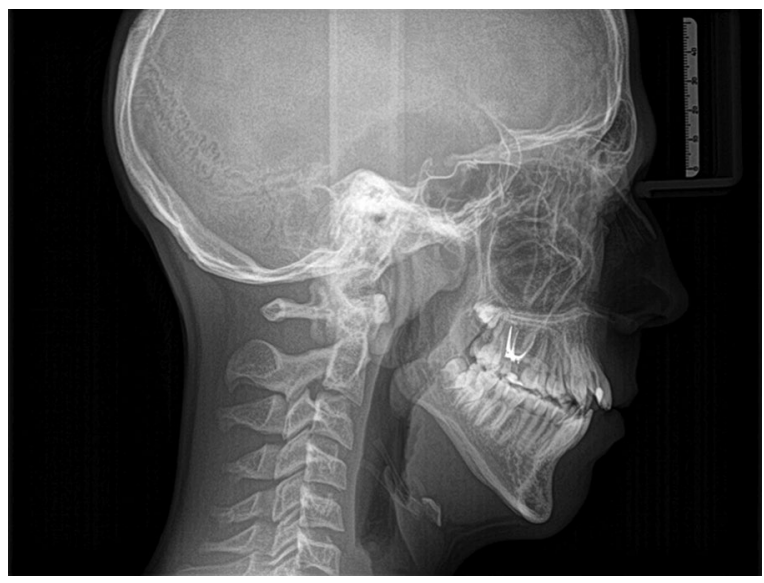

Figure 7. The increased soft tissue thickness in mentolabial sulcus area in subject with hyperdivergent pattern.

The discrepancy between our and other findings may be the result of racial differences, age group taken for the study and the size of the sample $[6,11]$.

The mentolabial sulcus area shows the opposite tendency compared to the other levels of measurement. This area increases the thickness going from hypo- towards hyperdivergent groups. According to Al Sajagh et al. [3], male hyperdivergent subjects have significantly larger soft tissue thickness at level B-Sm compared to normal and hypodivergent, which is similar to our results. This phenomenon of the increasing of the soft tissue thickness of mentolabial sulcus in hyperdivergent growth pattern, in which the majority of average thicknesses showed the smallest values, can be explained by the hypertrophy of the perioral musculature that tends to overcome vertical discrepancy and maintain lip competence (Fig. 7).

The results obtained by measuring the soft tissue chin thickness can be categorised into two groups. According to the first group, soft tissue chin thickness in all three levels (Pg-Pg1, Gn-Gn1 and Me-Me1) decreases by going from hypo- towards hyperdivergent group, and it is statistically significant only between these two groups $[5-8,22,24]$, which is only partly similar to our results. Namely, in the present study there is a significant difference between hypo- and hyperdivergent group at level Gn-Gn1, whereas at level Me-Me1 significant differences exist between normal and hyperdivergent group. At level Pg-Pg1, significant differences were not established, which was confirmed by another group of researchers $[9,15,20,21,23]$. They determined that hyperdivergent subjects have thinner soft tissues at level Gn-Gn1 
and Me-Me1, but not at level Pg-Pg1, the fact that they explained through the existence of differential extension between hard and soft tissues during growth. Shinde et al. [21] think that the area of pogonion is the least affected by (hyper) divergence. This is perhaps a natural manner to camouflage the existing state and give a more normal facial appearance. The reason that could account for the difference on menton between hyper- and normodivergent pattern may be the one that the soft tissue on menton apparently adjusts to the severe hyperdivergence, probably with the increased stretching of the soft tissue due to the increased divergence of the face. The finding that statistically significant difference happened between hyper- and hypodivergent patients emphasizes the fact that soft tissue thickness at menton is actually the thinnest of all distances in all groups. Our finding that soft tissue thickness at menton is minimal in hyperdivergent types of the face correlates with the research of Macari et al. [15] and Ashraf et al. [6]. Sodawala et al. [22] also did not report gender dimorphism for FSTT at all three levels of chin area, as opposed to the present study, where significant differences were established at level Me-Me1 in normodivergent group and at level Pg-Pg1 in hyperdivergent group. Somaiah et al. [23] were the only to publish the original result according to which females in hyperdivergent group had thicker FSTT than males at all tree levels, that is Pg-Pg1, Gn-Gn1 and Me-Me1.

The contrasting results of various studies suggest that the growth of soft tissue is different in individuals, different races and gender. The mechanism of compensational growth of soft tissue that happens only in one race may not happen in the other one [22]. The obtained results can be applied in forensic reconstruction of the face, orthognatic surgery and anthropology.

\section{CONCLUSIONS}

Facial soft tissue thickness showed a various degree of dependence on mandibular divergence pattern at different levels of measurement. The areas whose thickness is significantly conditioned by this pattern were established: the chin area at level Gn-Gn1, Me-Me1 and the region of the mentolabial sulcus (B-Sm). There are levels at which soft tissues get thinner from hypo- towards hyperdivergent group, but without statistical relevance, such as A-Sn and $\mathrm{N}-\mathrm{N} 1$, and there are levels on which vertical pattern has no influence, and that is the region of glabella and upper and lower lip thickness. At most levels, male subjects have thicker soft tissues and these differences are significant for all three groups in the subnasal area, whereas for some groups of divergence differences are significant in the area of lips, mentolabial sulcus and chin.

\section{Conflict of interest: None declared}

\section{REFERENCES}

1. Ahmed M, Shaikh A, Fida M. Diagnostic performance of various cephalometric parameters for the assessment of vertical growth pattern. Dental Press J Orthod. 2016; 21(4): 41-49, doi: 10.1590/2177-6709.21.4.041-049.oar, indexed in Pubmed: 27653263.

2. Ajwa N, Alkhars FA, AlMubarak FH, et al. Correlation between sex and facial soft tissue characteristics among young Saudi patients with various orthodontic skeletal malocclusions. Med Sci Monit. 2020; 26: e919771, doi: 10.12659/MSM.919771, indexed in Pubmed: 32101535.

3. Al-Sayagh NM, Saleem NR, Abdul-Qadir MY. Analysis of soft tissue facial profile in different vertical growth patterns. Al-Rafidain Dent J. 2011; 11(2): 346-356.

4. Al-Mashhadany S, Al-Chalabi H, Nadih M. Evaluation of facial soft tissue thickness in normal adults with different vertical discrepancies. Int J Sci Res. 2017; 6(2): 938-942, doi: 10.21275/ART2017603.

5. Anam S, Imtiaz A, Taskeen K. Assessment of the soft tissue chins thickness with different skeletal vertical patterns in Pakistani adults. J Dentistry Oral Hygiene. 2018; 10(1): 1-6, doi: 10.5897/jdoh2016.0192.

6. Ashraf K, Kulshrestha R, Azam A, et al. Soft tissue analysis of chin, upper lip length and thickness in patients with different mandibular divergent patterns: a cephalometric study. Indian J Orthodontics Dentofacial Res. 2020; 4(2): 88-93, doi: 10.18231/2455-6785.2018.0018.

7. Celikoglu M, Buyuk SK, Ekizer A, et al. Assessment of the soft tissue thickness at the lower anterior face in adult patients with different skeletal vertical patterns using cone-beam computed tomography. Angle Orthod. 2015; 85(2): 211-217, doi: 10.2319/040114-237.1, indexed in Pubmed: 24998911.

8. Cezairli N. Comparisons of soft tissue thickness measurements in adult patients with various vertical patterns. Meandros Med Dental J. 2017; 18(2): 120-129, doi: 10.4274/meandros.76376.

9. Feres $M$, Hitos $S$, Sousa $H$, et al. Comparação das dimensões de tecido mole entre padrões faciais distintos. Dental Press J Orthodontics. 2010; 15(4): 84-93, doi: 10.1590/s2176-94512010000400013.

10. Jazmati HM, Ajaj MA, Hajeer MY. Assessment of facial soft tissue dimensions in adult patients with different sagittal skeletal classes using cone beam computed tomography. J Contemp Dent Pract. 2016; 17(7): 542-548, indexed in Pubmed: 27595719.

11. Jeelani W, Fida M, Shaikh A, et al. Facial soft tissue thickness among various vertical facial patterns in adult Pakistani subjects. Forensic Sci Int. 2015; 257(1): 517.e1-517.e6, doi: 10.1016/j.forsciint.2015.09.006, indexed in Pubmed: 26476716. 
12. Kamak H, Celikoglu M. Facial soft tissue thickness among skeletal malocclusions: is there a difference? Korean J Orthod. 2012; 42(1): 23-31, doi: 10.4041/kjod.2012.42.1.23, indexed in Pubmed: 23112928.

13. Khare V, Niwlikar KB. Effect of vertical maxillary skelatal pattern on nasal morphology in high and low angle cases. Int J Oral Health Med Res. 2017; 3(6): 75-79.

14. Khatri JM, Sanap NB. Comparative evaluation of perioral soft tissue of skeletal normal Class I and Class II Division 1 subjects: A lateral cephalometric study. Int J Orthod Rehabil. 2020; 11(1): 1, doi: 10.4103/ijor.ijor_43_19.

15. Macari AT, Hanna AE. Comparisons of soft tissue chin thickness in adult patients with various mandibular divergence patterns. Angle Orthod. 2014; 84(4): 708-714, doi: 10.2319/062613-474.1, indexed in Pubmed: 24188123.

16. Meundi M, David C. Facial soft tissue thickness in South Indian adults with varied occlusions: a cone beam computed tomography study. J Indian Acad Oral Med Radiol. 2019; 31(3): 194, doi: 10.4103/jiaomr.jiaomr_83_19.

17. Oyonarte R, Hurtado M, Castro MV. Evolution of ANB and SN-GoGn angles during craniofacial growth: A retrospective longitudinal study. APOS Trends Orthod. 2016; 6: 295-301, doi: 10.4274/meandros.76376.

18. Perović T, Blažej Z. Male and female characteristics of facial soft tissue thickness in different orthodontic malocclusions evaluated by cephalometric radiography. Med Sci Monit. 2018; 24: 3415-3424, doi: 10.12659/MSM.907485, indexed in Pubmed: 29791323.

19. Ramesh G, Sreedhar NG, Narayanappa M. Facial soft tissue thickness in forensic facial reconstruction: is it enough if norms set? J Forensic Res. 2015; 06(05), doi: 10.4172/2157-7145.1000299.

20. Rasool G, Hussain T, Hussain U, et al. Comparisons of soft tissue chin thickness in adult patients with various mandibular divergence patterns. Pakistan Orthodontic J. 2016; 8(1): 53-57.

21. Shinde $N$, Jethe $S$, Agarkar $S$, et al. Comparative evaluation of soft tissue chin thickness in skeletal class $i$ and class II adults with three mandibular divergence: a cephalometric study. J Adv Med Dent Scie Res. 2019; 7(2): 33-40, doi: 10.21276/jamdsr.

22. Sodawala J, Akolkar A, Sodawala F, et al. Comparison of soft tissue chin thickness at different levels of chin in subjects with various growth patterns. Indian J Dent Res. 2020; 31(2): 224-228, doi: 10.4103/ijdr.IJDR_389_17, indexed in Pubmed: 32436901.

23. Khan MU, Somaiah S, Muddaiah S, et al. Comparison of soft tissue chin thickness in adult patients with various mandibular divergence patterns in Kodava population. Int J Orthod Rehabil. 2017; 8(2): 51, doi: 10.4103/ijor. ijor_38_16.

24. Subramaniam S, Karthi M, Kumar K, et al. Comparison of soft tissue chin prominence in various mandibular divergence patterns of Tamil Nadu population. J Indian Acad Dent Spec Res. 2016; 3(2): 39, doi: 10.4103/jiadsr. jiadsr_3_17.

25. Yemitan TA, Oludare YS, Ogunbanjo BO, et al. Vertical facial height and its correlation with skeletal pattern among young nigerian orthodontic patients. Int J Dentistry Oral Sci. 2018: 661-666, doi: 10.19070/2377-8075-18000130. 